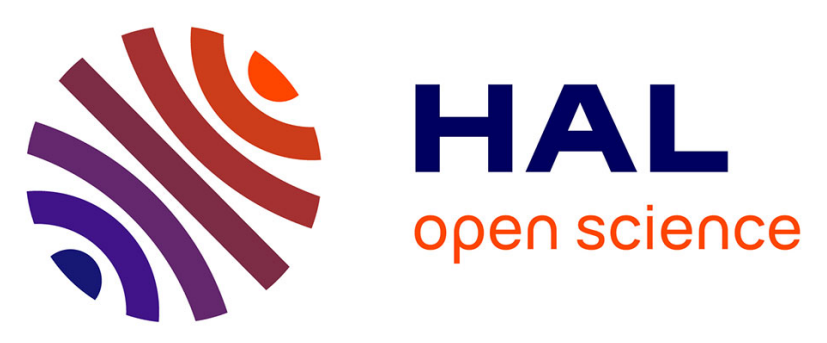

\title{
A parallel proximal splitting method for disparity estimation from multicomponent images under illumination variation
}

Caroline Chaux, Mireille El Gheche, Joumana Farah, Jean-Christophe

Pesquet, Beatrice Pesquet-Popescu

\section{To cite this version:}

Caroline Chaux, Mireille El Gheche, Joumana Farah, Jean-Christophe Pesquet, Beatrice PesquetPopescu. A parallel proximal splitting method for disparity estimation from multicomponent images under illumination variation. Journal of Mathematical Imaging and Vision, 2013, 47 (3), pp.167-178. 10.1007/s10851-012-0361-z . hal-00733454

\section{HAL Id: hal-00733454 \\ https://hal.science/hal-00733454}

Submitted on 19 Sep 2012

HAL is a multi-disciplinary open access archive for the deposit and dissemination of scientific research documents, whether they are published or not. The documents may come from teaching and research institutions in France or abroad, or from public or private research centers.
L'archive ouverte pluridisciplinaire HAL, est destinée au dépôt et à la diffusion de documents scientifiques de niveau recherche, publiés ou non, émanant des établissements d'enseignement et de recherche français ou étrangers, des laboratoires publics ou privés. 


\title{
A parallel proximal splitting method for disparity estimation from multicomponent images under illumination variation
}

\author{
C. Chaux - M. El-Gheche - J. Farah · J.-C. Pesquet • \\ B. Pesquet-Popescu
}

Received: date / Accepted: date

\begin{abstract}
Proximal splitting algorithms play a central role in finding the numerical solution of convex optimization problems. This paper addresses the problem of stereo matching of multi-component images by jointly estimating the disparity and the illumination variation. The global formulation being non-convex, the problem is addressed by solving a sequence of convex relaxations. Each convex relaxation is non trivial and involves many constraints aiming at imposing some regularity on the solution. Experiments demonstrate that the method is efficient and provides better results compared with other approaches.
\end{abstract}

\section{Chaux}

Université Paris-Est

Laboratoire d'Informatique Gaspard Monge - CNRS UMR 8049 77454 Marne la Vallée Cedex 2, France

E-mail: chaux@univ-mlv.fr

M. El-Gheche

Université Paris-Est

Laboratoire d'Informatique Gaspard Monge - CNRS UMR 8049

77454 Marne la Vallée Cedex 2, France

E-mail: el.gheche@univ-mlv.fr

J. Farah

Holy-Spirit University of Kaslik

Department of Telecommunications, Faculty of Engineering

P.O. Box 446, Jounieh, Lebanon

E-mail: joumanafarah@usek.edu.lb

J.-C. Pesquet

Université Paris-Est

Laboratoire d'Informatique Gaspard Monge - CNRS UMR 8049

77454 Marne la Vallée Cedex 2, France

E-mail: pesquet@univ-mlv.fr

B. Pesquet-Popescu

Telecom ParisTech,

Signal and Image Proc. Dept.

75014 Paris, France

E-mail: pesquet@telecom-paristech.fr
Keywords Stereo · disparity · color images · illumination variation $\cdot$ proximity operator $\cdot$ total variation tight frame $\cdot$ convex optimization $\cdot$ parallel proximal algorithms

\section{Introduction}

Depth estimation has become an increasingly important problem in computer vision as it constitutes a fundamental task in the reconstruction of realistic 3D scenes. A large number of algorithms have been proposed to perform this estimation. The problem being ill-posed, prior information (e.g. smoothness of the desired solution) needs to be taken into account in order to obtain reliable results.

In recent years, much progress has been made in stereo matching with the development of i) powerful global combinatorial optimization methods such as graph-cuts $[1,2]$ and belief propagation $[3,4]$; ii) variational approaches which have proven to be also very effective for globally solving the matching problem [5-7].

In a majority of dense disparity estimation techniques, the scene is assumed to be Lambertian. However, in the presence of illumination variation (that often occurs in practice), this assumption is violated. Several techniques have thus been proposed to either derive illumination invariant features or to model the illumination changes. Likewise, Cox et al. [8] proposed a dynamic histogram warping that consists of directly matching histogram values and performing a global optimization via dynamic programming. However, this approach is restricted to the case of a spatially invariant relationship between the intensities of the two images. Otherwise, methods based on non-parametric local transforms followed by normalized cross correla- 
tion (NCC) and rank-transform [9] have been used. In [10], a new invariant for stereo reconstruction called Light Transport Constancy was introduced to formulate a rank constraint and enable correspondence of non-Lambertian surfaces. The work in [11] shows that the intensities of two corresponding points are related by a spatially varying multiplicative term. Following this model, a stereo algorithm based on a nonconvex cost function was developed. The minimization of the cost function from the associated Euler-Lagrange equations becomes a difficult task. In the same context, based on this multiplicative model, dense disparity map estimation is formulated in [7] as a constrained optimization problem in which a strictly convex quadratic objective function is minimized under various convex constraints. The resulting optimization problem is solved via a parallel block iterative algorithm involving subgradient projections.

In this paper, we present an alternative strategy relying on a parallel proximal optimization approach [12]. Similarly to the subgradient based methods, we use the multiplicative model by Gennert which allows us to define a global similarity measure which is minimized over an intersection of convex constraint sets. The original contributions of our paper not only consist of the ability to consider multicomponent images in the presence of illumination variation, but also in the flexibility in minimizing various (possibly nonsmooth) convex similarity measures and combining them with various convex constraints.

The remaining of the paper is organized as follows. The notation, background and considered model are presented in section 2. Then, in section 3, we describe the adopted parallel proximal algorithm that allows us to solve the derived convex minimization problem. Some simulation results are shown in section 4 on the MiddleBury data set and other real images. Finally, some conclusions are drawn in section 5 .

\section{Stereo matching model}

We consider two multicomponent images of the same scene acquired by a stereoscopic camera. The left (resp. right) view is given by the function

$$
\begin{aligned}
I_{L}: & \mathbb{R}^{2} \mapsto \mathbb{R}^{K} \\
\mathbf{s} & \mapsto I_{L}(\mathbf{s})=\left(I_{L}^{(1)}(\mathbf{s}), I_{L}^{(2)}(\mathbf{s}), \ldots, I_{L}^{(K)}(\mathbf{s})\right)^{\top}
\end{aligned}
$$

(resp. $I_{R}: \mathbb{R}^{2} \mapsto \mathbb{R}^{K}$

$$
\left.\mathbf{s} \mapsto I_{R}(\mathbf{s})=\left(I_{R}^{(1)}(\mathbf{s}), I_{R}^{(2)}(\mathbf{s}), \ldots, I_{R}^{(K)}(\mathbf{s})\right)^{\top}\right) .
$$

For every $k \in\{1, \ldots, K\}, I_{R}^{(k)}(\mathbf{s})\left(\operatorname{resp} . I_{L}^{(k)}(\mathbf{s})\right)$ represents the $k$-th component of image $I_{R}$ (resp. $I_{L}$ ) at position $\mathbf{s} \in \mathbb{R}^{2}$. Particular cases of this framework include color images by taking $K=3$. Each component then corresponds to one of the color channels of a specific color system (RGB, YUV, ...). Throughout this paper, it will be assumed that $I_{R}$ and $I_{L}$ are differentiable functions.

Corresponding points in the images of the stereo pair tend to have similar values. The pixel at position $(x, y) \in \mathbb{R}^{2}$ in the left image $I_{L}$ corresponds to a pixel at position $\left(x^{\prime}, y^{\prime}\right) \in \mathbb{R}^{2}$ in the right image $I_{R}$, the disparity between these pixels being equal to $\left(x-x^{\prime}, y-y^{\prime}\right)$. When stereo images are rectified [13], the vertical component of the disparity vector vanishes. The disparity thus reduces to $u(x, y)=x-x^{\prime}$. Finding for each pixel $(x, y)$ in the left image the corresponding pixel $(x-u(x, y), y)$ in the right image constitutes the goal of stereo matching. The problem is then equivalent to finding a disparity field $u: \mathbb{R}^{2} \rightarrow[0,+\infty[$ which minimizes a similarity measure. However, under varying illumination conditions, the corresponding points in a stereo pair do not have the same component values. The illumination variation model we will employ is grounded on the work by Gennert [11] and takes the following form:

$\left(\forall(x, y) \in \mathbb{R}^{2}\right) \quad I_{R}(x-u(x, y), y) \simeq v(x, y) I_{L}(x, y)$

where $v: \mathbb{R}^{2} \rightarrow[0,+\infty[$ represents the illumination field. The problem now is not only to estimate the disparity $u$ but also to estimate the illumination field $v$. The next section aims at better formulating the considered problem.

\subsection{Problem formulation}

In what follows, we propose a variational approach to jointly estimate the disparity $u$ and the illumination variation $v$. Based on Model (2), we can formulate the stereo matching problem as the minimization of a similarity measure $\widetilde{J}$ :

$$
\begin{aligned}
& \widetilde{J}(\mathbf{u}, \mathbf{v})= \\
& \sum_{k=1}^{K} \sum_{\mathbf{s}=(x, y) \in \mathcal{A} \backslash \mathcal{O}} \phi^{(k)}\left(v(\mathbf{s}) I_{L}^{(k)}(\mathbf{s})-I_{R}^{(k)}(x-u(\mathbf{s}), y)\right)
\end{aligned}
$$

where, for every $k \in\{1, \ldots, K\}, \phi^{(k)}$ is assumed to belong to $\Gamma_{0}(\mathbb{R})$, the class of proper lower semi-continuous 
convex functions from $\mathbb{R}$ to $]-\infty,+\infty]$. In addition, $\mathcal{A} \subset \mathbb{Z}^{2}$ is the considered finite image domain, $\mathbf{u}=$ $(u(\mathbf{s}))_{\mathbf{s} \in \mathcal{A}}$, and $\mathbf{v}=(v(\mathbf{s}))_{\mathbf{s} \in \mathcal{A}}$. The occlusion areas denoted by $\mathcal{O}$ correspond to pixels only visible from one view of the stereo pair, which should not be taken into account in the computation of the similarity measure. Consequently, they have been discarded in the expression of the cost function.

In this paper, we will be mainly concerned with convex optimization approaches. Unfortunately, $\widetilde{J}$ is nonconvex with respect to the variable $\mathbf{u}$. To tackle this difficulty, we perform the first-order Taylor expansion of the disparity compensated right image around an initial value of the disparity $\overline{\mathbf{u}}$. The latter can be derived from a rough estimation, e.g. by a block-based correlation method [14]. In practice, we can iteratively update our initial value in order to make our final solution weakly dependent on it. When the magnitude of the difference of the fields $\mathbf{u}$ and $\overline{\mathbf{u}}=(\bar{u}(\mathbf{s}))_{\mathbf{s} \in \mathcal{A}}$ is small enough, we obtain the following expression: for every $k \in\{1, \ldots, K\}$ and $\mathbf{s}=(x, y) \in \mathcal{A}$,

$$
\begin{aligned}
& I_{R}^{(k)}(x-u(\mathbf{s}), y) \simeq I_{R}^{(k)}(x-\bar{u}(\mathbf{s}), y) \\
&-(u(\mathbf{s})-\bar{u}(\mathbf{s})) \nabla^{(1)} I_{R}^{(k)}(x-\bar{u}(\mathbf{s}), y)
\end{aligned}
$$

where $\nabla^{(1)} I_{R}^{(k)}$ denotes the horizontal gradient of the $k$-th component of the right image.

As a consequence of (3) and (4), we can approximate the cost function by:

$$
\begin{aligned}
& J(\mathbf{u}, \mathbf{v}) \\
& =\sum_{k=1}^{K} \sum_{s \in \mathcal{A} \backslash \mathcal{O}} \phi^{(k)}\left(T_{1}^{(k)}(\mathbf{s}) u(\mathbf{s})+T_{2}^{(k)}(\mathbf{s}) v(\mathbf{s})-r^{(k)}(\mathbf{s})\right)
\end{aligned}
$$

where, for every $k \in\{1, \ldots, K\}$ and $\mathbf{s}=(x, y) \in \mathcal{A}$,

$$
\left\{\begin{array}{l}
T_{1}^{(k)}(\mathbf{s})=\nabla^{(1)} I_{R}^{(k)}(x-\bar{u}(\mathbf{s}), y) \\
T_{2}^{(k)}(\mathbf{s})=I_{L}^{(k)}(\mathbf{s}) \\
r^{(k)}(\mathbf{s})=I_{R}^{(k)}(x-\bar{u}(\mathbf{s}), y)+\bar{u}(\mathbf{s}) T_{1}^{(k)}(\mathbf{s}) .
\end{array}\right.
$$

As previously mentioned, our objective is to jointly estimate $\mathbf{u}$ and $\mathbf{v}$. Thus, by defining $\mathbf{w}=(\mathbf{u}, \mathbf{v})$,

$$
(\forall \mathbf{s} \in \mathcal{A}) \quad w(\mathbf{s})=\left[\begin{array}{l}
u(\mathbf{s}) \\
v(\mathbf{s})
\end{array}\right]
$$

and, for every $k \in\{1, \ldots, K\}$ and $\mathbf{s} \in \mathbb{R}^{2}, \mathbf{T}^{(k)}(\mathbf{s})=$ $\left[T_{1}^{(k)}(\mathbf{s}), T_{2}^{(k)}(\mathbf{s})\right]$, the above expression can be reexpressed more concisely as

$$
J(\mathbf{w})=\sum_{k=1}^{K} J^{(k)}(\mathbf{w})
$$

where, for every $k \in\{1, \ldots, K\}$,

$$
J^{(k)}(\mathbf{w})=\sum_{s \in \mathcal{A} \backslash \mathcal{O}} \phi^{(k)}\left(\mathbf{T}^{(k)}(\mathbf{s}) w(\mathbf{s})-r^{(k)}(\mathbf{s})\right) .
$$

Despite the convexity of the function $J$, optimizing this criterion is an ill-posed problem, since we have two variables to estimate at each point $\mathbf{s}$ and the components of $T^{(k)}(\mathbf{s})$ may locally vanish for some $k$ and $\mathbf{s}$. We thus need to incorporate additional prior information on the desired disparity and illumination variation fields.

\subsection{Introducing prior information}

Our objective here is to introduce prior information on our target solution. This can be done either by adding to $J$ some regularization terms or by incorporating some convex constraints to the problem. In this paper, we will follow the second approach. Let $\left(S_{i}\right)_{1 \leq i \leq m}$ denote the $m$ nonempty closed convex sets modelling the constraints one wants to impose. The resulting optimization problem can then be formulated as:

Find $\mathbf{w} \in S=\bigcap_{i=1}^{m} S_{i}$ such that $J(\mathbf{w})=\inf J(S)$.

Constraint sets defined on the Hilbert space $\mathcal{H}=$ $\mathbb{R}^{|\mathcal{A}|} \times \mathbb{R}^{|\mathcal{A}|}$ can be described as lower level sets:

$(\forall i \in\{1, \ldots, m\}) \quad S_{i}=\left\{\mathbf{w} \in \mathcal{H} \mid f_{i}(\mathbf{w}) \leq \delta_{i}\right\}$,

where, for every $i \in\{1, \ldots, m\}, f_{i}: \mathcal{H} \rightarrow \mathbb{R}$ is a convex function and $\delta_{i} \in \mathbb{R}$. One of the potential advantages of this approach with respect to the regularization formulation is that it appears often easier from a physical viewpoint to set the constraint bounds than determining regularization parameters.

We will now review some of the constraints that can be applied to our problem.

\subsubsection{Constraints that can be applied on the disparity}

Range values As the minimum and maximum values $u_{\min }$ and $u_{\max }$ of the disparity field are often known, we propose to introduce a constraint related to these bounds. The corresponding constraint can be expressed as

$$
\begin{aligned}
S_{1,1}=\left\{(u(\mathbf{s}))_{\mathbf{s} \in \mathcal{A}}\right. & \in \mathbb{R}^{|\mathcal{A}|} \mid \\
& \left.(\forall \mathbf{s} \in \mathcal{A}) \quad u_{\min } \leq u(\mathbf{s}) \leq u_{\max }\right\}
\end{aligned}
$$

It can be noticed that the disparity is always nonnegative, so that $u_{\min } \geq 0$. 
First-order smoothness constraint A piecewise constant behaviour of the disparity field is often expected. So, it is meaningful to introduce a constraint enforcing smoothness in homogeneous areas while preserving edges. One can think of using the Total Variation semi-norm which has played a key role in image recovery problems [15, 16]. Let $\widehat{\nabla^{(1)}}$ and $\widehat{\nabla^{(2)}}$ denote discrete horizontal and vertical gradients (for example, obtained by cyclic convolutions $[17,18])$. Then, a discrete version of the total variation $(\mathrm{TV})$ is the following one:

$$
\begin{aligned}
& \left(\forall \mathbf{u} \in \mathbb{R}^{|\mathcal{A}|}\right) \\
& \operatorname{TV}(\mathbf{u})=\sum_{\mathbf{s} \in \mathcal{A}} \sqrt{\left|\widehat{\nabla^{(1)}} u(\mathbf{s})\right|^{2}+\left|\widehat{\nabla^{(2)}} u(\mathbf{s})\right|^{2}} .
\end{aligned}
$$

The associated constraint set is

$S_{1,2}=\left\{\mathbf{u} \in \mathbb{R}^{|\mathcal{A}|} \mid \mathrm{TV}(\mathbf{u}) \leq \tau_{2}\right\}$

with $\tau_{2}>0$.

Frame analysis constraint Alternatively, we can adopt a frame analysis approach to construct a smoothness constraint. In particular, it well-known $[19,20]$ that wavelet frames, through their relationships with Besov spaces, constitute appropriate tools to characterize useful classes of regular signals. Frame representations [21] and more precisely tight frame representations have become very popular during the last decade. Such transforms can be described by an analysis frame operator $F: \mathbb{R}^{|\mathcal{A}|} \rightarrow \mathbb{R}^{Q}$ with $Q \geq|\mathcal{A}|$, which associates to $\mathbf{u} \in \mathbb{R}^{|\mathcal{A}|}$ its frame coefficients $\left((F \mathbf{u})_{q}\right)_{1 \leq q \leq Q}$. The considered frame analysis constraint is expressed as

$S_{1,2}^{\prime}=\left\{\mathbf{u} \in \mathbb{R}^{|\mathcal{A}|}\left|\sum_{q=1}^{Q} \eta_{q}\right|(F \mathbf{u})_{q} \mid \leq \tau_{2}^{\prime}\right\}$

where $\left(\eta_{q}\right)_{1 \leq q \leq Q} \in\left[0,+\infty\left[^{Q}\right.\right.$ and $\tau_{2}^{\prime}>0 . F$ is said to be a tight frame when $F^{\top} F=\nu I$, where $\nu>0$. A simple example of a tight frame is the union of $\nu$ orthonormal wavelet bases [22].

Second-order constraint Some recent works [23] have shown that considering the second-order derivatives of the target image can be helpful in data recovery problems. Let a discrete version of the Hessian of $\mathbf{u} \in \mathbb{R}^{|\mathcal{A}|}$ at $\mathbf{s} \in \mathcal{A}$ be

$\widehat{\nabla^{2}} \mathbf{u}(\mathbf{s})=\left[\begin{array}{ll}\widehat{\nabla^{(1,1)}} u(\mathbf{s}) & \widehat{\nabla^{(1,2)}} u(\mathbf{s}) \\ \widehat{\nabla^{(1,2)}} u(\mathbf{s}) & \widehat{\nabla^{(2,2)}} u(\mathbf{s})\end{array}\right]$
Consequently, a second-order variant of the discrete total variation can be defined as

$$
\begin{aligned}
& \left(\forall \mathbf{u} \in \mathbb{R}^{|\mathcal{A}|}\right) \quad \operatorname{TV}_{2}(\mathbf{u})= \\
& \quad \sum_{\mathbf{s} \in \mathcal{A}} \sqrt{\left|\widehat{\nabla^{(1,1)}} u(\mathbf{s})\right|^{2}+\left|\widehat{\left.\nabla^{2,2}\right)} u(\mathbf{s})\right|^{2}+2\left|\widehat{\nabla^{(1,2)}} u(\mathbf{s})\right|^{2}}
\end{aligned}
$$

and the associated constraint set is

$S_{1,3}=\left\{\mathbf{u} \in \mathbb{R}^{|\mathcal{A}|} \mid \mathrm{TV}_{2}(\mathbf{u}) \leq \tau_{3}\right\}$

with $\tau_{3}>0$

\subsubsection{Constraints that can be applied on the illumination field}

Range values Similarly to the disparity field, estimations of the minimum and maximum values $v_{\text {min }} \geq 0$ and $v_{\max }$ of the illumination field are often available. The corresponding constraint can be expressed as

$$
\begin{aligned}
S_{2,1}=\left\{(v(\mathbf{s}))_{\mathbf{s} \in \mathcal{A}}\right. & \in \mathbb{R}^{|\mathcal{A}|} \mid \\
& \left.(\forall \mathbf{s} \in \mathcal{A}) \quad v_{\min } \leq v(\mathbf{s}) \leq v_{\max }\right\} .
\end{aligned}
$$

First-order smoothness constraint Since the illumination field is usually smoothly varying, we can add a quadratic constraint on the discrete gradient of the illumination field. This amounts to considering the following constraint set:

$S_{2,2}=\left\{\mathbf{v} \in \mathbb{R}^{|\mathcal{A}|} \mid\|\widehat{\nabla} \mathbf{v}\|_{\ell_{2}}^{2} \leq \kappa_{2}\right\}$

where $\kappa_{2}>0$ and

$\left(\forall \mathbf{v} \in \mathbb{R}^{|\mathcal{A}|}\right) \quad\|\widehat{\nabla} \mathbf{v}\|_{\ell^{2}}=\left(\sum_{\mathbf{s} \in \mathcal{A}}\left|\widehat{\nabla^{(1)}} v(\mathbf{s})\right|^{2}+\left|\widehat{\nabla^{(2)}} v(\mathbf{s})\right|^{2}\right)^{1 / 2}$

Second-order constraint We can also introduce a constraint on the discrete Hessian of the illumination field, thus yielding

$$
S_{2,3}=\left\{\mathbf{v} \in \mathbb{R}^{|\mathcal{A}|} \mid\left\|\widehat{\nabla^{2}} \mathbf{v}\right\|_{\ell_{2}}^{2} \leq \kappa_{3}\right\}
$$

where $\kappa_{3}>0$ and, for every $\mathbf{v} \in \mathbb{R}^{|\mathcal{A}|}$,

$$
\begin{array}{r}
\left\|\widehat{\nabla^{2}} \mathbf{v}\right\|_{\ell^{2}}=\left(\sum_{\mathbf{s} \in \mathcal{A}}\left|\widehat{\nabla^{(1,1)}} v(\mathbf{s})\right|^{2}+\left|\widehat{\nabla^{(2,2)}} v(\mathbf{s})\right|^{2}\right. \\
\left.+2\left|\widehat{\nabla^{(1,2)}} v(\mathbf{s})\right|^{2}\right)^{1 / 2}
\end{array}
$$




\subsubsection{Resulting constraints applied on vector $\mathbf{w}$}

Based on the constraints presented above, four different constraint sets are considered in this work:

1. The first one concerns the range values taken by the disparity and the illumination fields. It is defined as

$$
S_{1}=S_{1,1} \times S_{2,1}
$$

2. The second set is based on the first-order regularity of the illumination and disparity fields:

$$
S_{2}=S_{1,2} \times S_{2,2}
$$

3. The third set involves the frame analysis formulation instead of the total variation for the disparity, and reads:

$$
S_{2}^{\prime}=S_{1,2}^{\prime} \times S_{2,2}
$$

4. The last constraint set models the second-order smoothness constraints:

$$
S_{3}=S_{1,3} \times S_{2,3}
$$

\section{Proximal approaches for convex optimization}

\subsection{Proximity operator}

In a seminal paper [24], Moreau proposed an extension of the notion of projection by considering the following optimization problem:

$$
\underset{z \in \mathcal{H}}{\operatorname{minimize}} \quad \varphi(z)+\frac{1}{2}\|z-y\|^{2}
$$

where $\mathcal{H}$ is a real Hilbert space, $\varphi \in \Gamma_{0}(\mathcal{H})$ and $y \in \mathcal{H}$. This problem admits a unique solution which is called the proximity operator $\operatorname{prox}_{\varphi} y$ of $\varphi$ at $y$.

Consequently, by taking $\varphi=\iota_{C}$, where the indicator function $\iota_{C}$ of a nonempty closed convex set $C \subset \mathcal{H}$ is defined as

$$
(\forall z \in \mathcal{H}) \quad \iota_{C}(z)= \begin{cases}0 & \text { if } z \in C \\ +\infty & \text { otherwise }\end{cases}
$$

$\operatorname{prox}_{\iota_{C}}$ reduces to the projection operator $P_{C}$ onto $C$ [25-27].

Proximity operators have very attractive properties [26] that make them particularly well-suited to design iterative minimization algorithms.

\subsection{Optimization background}

The constraints proposed in section 2.2.3 are separable since each constraint set $S_{i}$ with $i \in\{1, \ldots, m\}$ can be expressed as $S_{1, i} \times S_{2, i}{ }^{1}$ In addition, for every $i \in\{1, \ldots, m\}$, one can express $S_{1, i}$ (resp. $S_{2, i}$ ) as $L_{1, i}^{-1}\left(C_{1, i}\right)$ (resp. $\left.L_{2, i}^{-1}\left(C_{2, i}\right)\right)$ where $C_{1, i}\left(\right.$ resp. $\left.C_{2, i}\right)$ is a nonempty closed convex subset of $\mathbb{R}^{N_{1, i}}$ (resp. $\mathbb{R}^{N_{2, i}}$ ) and $L_{1, i}$ (resp. $L_{2, i}$ ) is a matrix in $\mathbb{R}^{N_{1, i} \times|\mathcal{A}|}$ (resp. $\left.\mathbb{R}^{N_{2, i} \times|\mathcal{A}|}\right)$. For example, we have chosen $L_{1,1}$ and $L_{2,1}$ equal to the $|\mathcal{A}| \times|\mathcal{A}|$ identity matrix. By setting $L_{1,2}$ and $L_{2,2}$ equal to the concatenation of the horizontal and vertical gradient operators $\left(N_{1,2}=N_{2,2}=2|\mathcal{A}|\right)$, $C_{1,2}$ and $C_{2,2}$ reduce to $\ell_{2,1}$ balls $[28,29]$. A similar choice can be made for constraint $S_{3}$ by considering linear operators related to the second-order derivatives $\left(N_{1,3}=N_{2,3}=3|\mathcal{A}|\right)$. In the considered cases, the projection onto each convex set $C_{1, i}$ or $C_{2, i}$ takes a closed form or it can be computed in a finite number of operations [28]. The considered optimization problem then takes the following generic form:

$$
\begin{aligned}
& \underset{1, i \mathbf{u} \in C_{1, i}, L_{2, i} \mathbf{v} \in C_{2, i},}{\operatorname{minimize}} J(\mathbf{w}) . \\
& i \in\{1, \ldots, m\}
\end{aligned}
$$

Numerical solutions to this problem can be provided by parallel proximal splitting methods. These methods basically consist of iterating computations of proximity operators.

\subsection{PPXA + algorithm}

In this work, we employ the Parallel Proximal Algorithm $(\mathrm{PPXA}+)$ [12]. This algorithm is closely related to augmented Lagrangian approaches [30,31]. The existing links have been investigated in [12, Section 6] where it is shown that the parallel versions of the alternating direction method of multipliers in [31,32] constitute a particular case of PPXA + . Alternative algorithms could be employed in our context [33-38]. However, PPXA + was observed to have a good convergence profile with respect to other competing algorithms.

As shown by Algorithm 1, we proceed by splitting function $J$ in the $K$ terms corresponding to each image component and by exploiting the separability of the closed convex constraint sets $\left(C_{1, i} \times C_{2, i}\right)_{1 \leq i \leq m}$. The algorithm is initialized by setting the positive weights $\left.\left(\omega_{1}, \omega_{2}, \ldots, \omega_{m+K}\right) \in\right] 0,+\infty\left[^{m+K}\right.$ as well as the variables $\left(\mathbf{z}_{1, i, 0}\right)_{1 \leq i \leq m+K}$ and $\left(\mathbf{z}_{2, i, 0}\right)_{1 \leq i \leq m+K}$ associated

1 Constraint $S_{2}^{\prime}$ will be substituted for $S_{2}$ in some of our experiments. 
with the convex constraints $\left(C_{1,1} \times C_{2,1}, \ldots, C_{1, m} \times\right.$ $\left.C_{2, m}\right)$ and the split data fidelity criterion $J \mapsto \mathbf{w} \mapsto\left(J^{(1)}(\mathbf{w}), \ldots, J^{(K)}(\mathbf{w})\right)$.

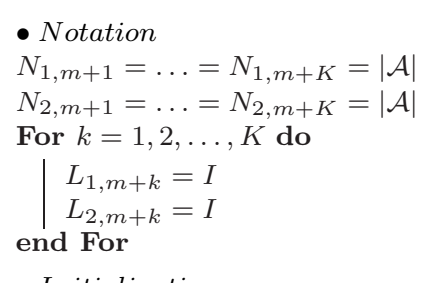

\section{- Initialization}

$\left.\left(\omega_{1}, \omega_{2}, \ldots, \omega_{m+K}\right) \in\right] 0,+\infty\left[^{m+K}\right.$

$\left(\mathbf{z}_{1, i, 0}\right)_{1 \leq i \leq m+K} \in \mathbb{R}^{N_{1,1}} \times \mathbb{R}^{N_{1,2}} \times \cdots \times \mathbb{R}^{N_{1, m+K}}$

$\left(\mathbf{z}_{2, i, 0}\right)_{1 \leq i \leq m+K} \in \mathbb{R}^{N_{2,1}} \times \mathbb{R}^{N_{2,2}} \times \cdots \times \mathbb{R}^{N_{2, m+K}}$

$Q_{1}=\left(\sum_{i=1}^{m+K} \omega_{i}\left(L_{1, i}\right)^{\top} L_{1, i}\right)^{-1}$

$Q_{2}=\left(\sum_{i=1}^{m+K} \omega_{i}\left(L_{2, i}\right)^{\top} L_{2, i}\right)^{-1}$

$\mathbf{u}_{0}=Q_{1}\left(\sum_{i=1}^{m+K} \omega_{i}\left(L_{1, i}\right)^{\top} \mathbf{z}_{1, i, 0}\right)$

$\mathbf{v}_{0}=Q_{2}\left(\sum_{i=1}^{m+K} \omega_{i}\left(L_{2, i}\right)^{\top} \mathbf{z}_{2, i, 0}\right)$

- Main loop

For $n=0,1, \ldots$ do

- Direct projections

For $i=1,2, \ldots, m$ do

$\mid \mathbf{p}_{1, i, n}=P_{C_{1, i}}\left(\mathbf{z}_{1, i, n}\right)$ and $\mathbf{p}_{2, i, n}=P_{C_{2, i}}\left(\mathbf{z}_{2, i, n}\right)$ end For

- Prox computations

For $k=1,2, \ldots, K$ do

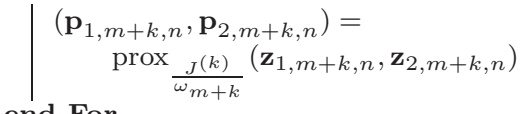

end For

- Averaging

$\mathbf{c}_{1, n}=Q_{1}\left(\sum_{i=1}^{m+K} \omega_{i}\left(L_{1, i}\right)^{\top} \mathbf{p}_{1, i, n}\right)$

$\mathbf{c}_{2, n}=Q_{2}\left(\sum_{i=1}^{m+K} \omega_{i}\left(L_{2, i}\right)^{\top} \mathbf{p}_{2, i, n}\right)$

\section{- Updates}

For $i=1,2, \ldots, m$ do

$\mathbf{z}_{1, i, n+1}=\mathbf{z}_{1, i, n}+\lambda_{n}\left(L_{1, i}\left(2 \mathbf{c}_{1, n}-\mathbf{u}_{n}\right)-\mathbf{p}_{1, i, n}\right)$

$\mathbf{z}_{2, i, n+1}=\mathbf{z}_{2, i, n}+\lambda_{n}\left(L_{2, i}\left(2 \mathbf{c}_{2, n}-\mathbf{v}_{n}\right)-\mathbf{p}_{2, i, n}\right)$

end For

For $k=1,2, \ldots, K$ do

$\mathbf{z}_{1, m+k, n+1}=\mathbf{z}_{1, m+k, n}+\lambda_{n}\left(2 \mathbf{c}_{1, n}-\mathbf{u}_{n}-\right.$

$\left.\mathbf{p}_{1, m+k, n}\right)$

$\mathbf{z}_{2, m+k, n+1}=\mathbf{z}_{2, m+k, n}+\lambda_{n}\left(2 \mathbf{c}_{2, n}-\mathbf{v}_{n}-\right.$ $\left.\mathbf{p}_{2, m+k, n}\right)$

end For

$\mathbf{u}_{n+1}=\mathbf{u}_{n}+\lambda_{n}\left(\mathbf{c}_{1, n}-\mathbf{u}_{n}\right)$

$\mathbf{v}_{n+1}=\mathbf{v}_{n}+\lambda_{n}\left(\mathbf{c}_{2, n}-\mathbf{v}_{n}\right)$

end For eling prior information. By using basic properties of proximity operators [26], it can be shown that, for every $k \in\{1, \ldots, K\}, \omega_{k}>0$, and $\left(\mathbf{z}_{1}, \mathbf{z}_{2}\right) \in \mathbb{R}^{|\mathcal{A}|} \times \mathbb{R}^{|\mathcal{A}|}$,

$\operatorname{prox}_{\frac{J(k)}{\omega_{k}}}\left(\mathbf{z}_{1}, \mathbf{z}_{2}\right)$

$=\left(z_{1}(\mathbf{s})+\mu^{(k)}(\mathbf{s}) T_{1}^{(k)}(\mathbf{s}), z_{2}(\mathbf{s})+\mu^{(k)}(\mathbf{s}) T_{2}^{(k)}(\mathbf{s})\right)_{\mathbf{s} \in \mathcal{A}}$

where $\mathbf{z}_{1}=\left(z_{1}(\mathbf{s})\right)_{\mathbf{s} \in \mathcal{A}}, \mathbf{z}_{2}=\left(z_{2}(\mathbf{s})\right)_{\mathbf{s} \in \mathcal{A}}$, and

$$
\mu^{(k)}(\mathbf{s})= \begin{cases}\frac{1}{\gamma^{(k)}(\mathbf{s})}\left(\operatorname{prox}_{\frac{\gamma^{(k)}(\mathbf{s}) \phi^{(k)}}{\omega_{k}}}\left(\tau^{(k)}(\mathbf{s})\right)-\tau^{(k)}(\mathbf{s})\right) \\ \text { if } \gamma^{(k)}(\mathbf{s}) \neq 0 \\ \text { and } \mathbf{s} \notin \mathcal{O} \\ \text { otherwise }\end{cases}
$$

$\tau^{(k)}(\mathbf{s})=T_{1}^{(k)}(\mathbf{s}) z_{1}(\mathbf{s})+T_{2}^{(k)}(\mathbf{s}) z_{2}(\mathbf{s})-r^{(k)}(\mathbf{s})$

$\gamma^{(k)}(\mathbf{s})=\left|T_{1}^{(k)}(\mathbf{s})\right|^{2}+\left|T_{2}^{(k)}(\mathbf{s})\right|^{2}$.

Subsequently, an averaging of the variables resulting from the computation of the projections and proximity operators is performed before the update equations. It must be emphasized that the matrix inversions required in the algorithm can be efficiently performed by making use of discrete Fourier diagonalization techniques [39]. To gain insights in the convergence properties of this algorithm, assume that, for every $k \in\{1, \ldots, K\}, \phi^{(k)}$ is a finite function. In our problem, the data fidelity term is convex with respect to the variable $(\mathbf{u}, \mathbf{v})$ and each individual constraint is also convex with respect to either $\mathbf{u}$ or $\mathbf{v}$, as mentioned in sections 2.1 and 2.2. Then, provided that the sequence of relaxation parameters $\left(\lambda_{n}\right)_{n \in \mathbb{N}}$ involved in Algorithm 1 is such that $(\forall n \in \mathbb{N})$ $\tilde{\lambda} \leq \lambda_{n+1} \leq \lambda_{n}<2$, where $\left.\tilde{\lambda} \in\right] 0,2[$, [12, Prop. 5.2] allows us to guarantee that the sequence $\left(\mathbf{u}_{n}, \mathbf{v}_{n}\right)_{n \in \mathbb{N}}$ generated by the algorithm converges to a solution to problem (10). The value of this parameter is set to 1.5 for all iterations. As the relaxation parameter constitutes an internal parameter of the algorithm, its choice does not modify the optimality of the solution. However, its value plays a role in the convergence speed as illustrated in Table 1. According to this table, the value of $\lambda_{n}$ has been chosen so as to maximize the convergence speed.

Note finally that PPXA + can be similarly applied in order to minimize $J$ over $S_{1} \cap S_{2}$ or $S_{1} \cap S_{3}$.

Algorithm 1: PPXA+

\section{Results}

\subsection{Error measures}

First of all, we would like to define the quality measures

we adopt. Let $\widehat{z} \in \mathbb{R}^{|\mathcal{A}|}$ denote an estimate of $z \in \mathbb{R}^{|\mathcal{A}|}$. imity operators of the data fidelity terms, as well as the direct projections onto the different convex sets mod- 


\begin{tabular}{|l|l|l|l|l|l|l|l|l|l|l|l|}
\hline$\lambda_{n}$ & 0.1 & 0.3 & 0.5 & 0.7 & 0.9 & 1 & 1.1 & 1.3 & $\mathbf{1 . 5}$ & 1.7 & 1.9 \\
\hline Teddy & 90.79 & 30.27 & 18.16 & 12.97 & 10.09 & 9.09 & 8.26 & 6.98 & $\mathbf{6 . 2 9}$ & 6.77 & 7.35 \\
\hline Cones & 98.07 & 32.75 & 19.69 & 14.08 & 10.96 & 9.89 & 9.01 & 10.00 & $\mathbf{9 . 0 5}$ & 10.55 & 11.07 \\
\hline
\end{tabular}

Table 1 Influence of $\lambda_{n}$ on execution time (in seconds) for PPXA + algorithm. The stopping condition described in section 4.3 is used.

The measures below allow us to provide quantitative evaluations of our results:

1. Mean Absolute Error (MAE)

$$
\operatorname{MAE}(z, \widehat{z})=\frac{1}{|\mathcal{A} \backslash \mathcal{O}|} \sum_{\mathbf{s} \in \mathcal{A} \backslash \mathcal{O}}|z(\mathbf{s})-\hat{z}(\mathbf{s})|
$$

2. Average percentage of bad pixels

$$
\operatorname{Err}(z, \widehat{z})=\frac{1}{|\mathcal{A} \backslash \mathcal{O}|} \sum_{\mathbf{s} \in \mathcal{A} \backslash \mathcal{O}} 1_{\{|z(\mathbf{s})-\hat{z}(\mathbf{s})|>T\}}
$$

where $T$ is some given positive threshold value chosen here equal to 2 and, for every $\mathbf{s} \in \mathcal{A}$,

$$
1_{\{|z(\mathbf{s})-\hat{z}(\mathbf{s})|>T\}}= \begin{cases}1 & \text { if }|z(\mathbf{s})-\hat{z}(\mathbf{s})|>T \\ 0 & \text { otherwise }\end{cases}
$$

\subsection{Initialization}

As previously mentioned, initial estimates $\overline{\mathbf{u}}$ and $\overline{\mathbf{v}}$ have been obtained based on a block matching technique. Usually, two criteria are considered in the minimization performed in the block matching procedure: the sum of squared differences and the sum of absolute differences. In this work, we consider another popular measure, namely the Normalized Cross Correlation (NCC) similarity measure [14]. Note that more sophisticated measures could be envisaged such as the one proposed in [40]. NCC is defined as follows:

$$
(\forall(x, y) \in \mathcal{A})(\forall u \in \mathbb{N}) \quad \psi_{x, y}(u)=\sum_{k=1}^{K} \psi_{x, y}^{(k)}(u)
$$

where

$$
\begin{aligned}
& \psi_{x, y}^{(k)}(u)= \\
& \frac{\sum_{(i, j) \in \mathcal{B}} I_{L}^{(k)}(x+i, y+j) I_{R}^{(k)}(x-u+i, y+j)}{\sqrt{\sum_{(i, j) \in \mathcal{B}}\left(I_{L}^{(k)}(x+i, y+j)\right)^{2}} \sqrt{\sum_{(i, j) \in \mathcal{B}}\left(I_{R}^{(k)}(x-u+i, y+j)\right)^{2}}}
\end{aligned}
$$

and $\mathcal{B}$ corresponds to the matching block (here of size $5 \times 5)$ centered at pixel $(x, y)$ in the reference image.

In order to get the corresponding pixel $(x-\bar{u}(x, y), y)$ in the right image, the matching process determines $\bar{u}(x, y)$ which maximizes the similarity measure $\psi_{x, y}$ :

$$
\bar{u}(x, y) \in \underset{u \in \mathbb{N} \cap\left[u_{\min }, u_{\max }\right]}{\operatorname{argmax}} \psi_{x, y}(u) .
$$

Once $\bar{u}(x, y)$ is found, $\bar{v}(x, y)$ is evaluated by the following weighted least squares estimation:

$$
\begin{aligned}
& \bar{v}(x, y)= \\
& \frac{\sum_{k=1}^{K} \theta^{(k)} \sum_{(i, j) \in \mathcal{B}} I_{L}^{(k)}(x+i, y+j) I_{R}^{(k)}(x-\bar{u}(x, y)+i, y+j)}{\sum_{k=1}^{K} \theta^{(k)} \sum_{(i, j) \in \mathcal{B}} I_{L}^{(k)}(x+i, y+j)^{2}}
\end{aligned}
$$

where $\left(\theta^{(k)}\right)_{1 \leq k \leq K}$ are nonnegative real weights. In our tests, the illumination variation is the same for all the components and, for YUV images, the best results have been obtained by choosing a weight equal to 1 for the $\mathrm{Y}$ component, and 0 for the $\mathrm{U}$ and $\mathrm{V}$ components.

However, with the previous technique, some remaining artefacts can be observed in the disparity maps, e.g. contouring effects and oversmoothing in some areas. In order to reduce these artifacts and limit the influence of occlusion areas, a bidirectional matching process is performed. More precisely, we firstly apply the NCC method to find the left disparity map denoted by $\bar{u}_{L}(x, y)$, by proceeding as described above. Similarly, we apply the NCC method to find the right disparity map denoted by $\bar{u}_{R}(x, y)$ by taking now the right view as the reference image. Finally, we derive the initial disparity $\bar{u}(x, y)$ as follows:

$\bar{u}(x, y)=\bar{u}_{R}\left(x-\bar{u}_{L}(x, y), y\right)$.

The upper and lower bounds in the constrained formulation are estimated using prior knowledge when available or from the initial estimates $\overline{\mathbf{u}}$ and $\overline{\mathbf{v}}$, otherwise.

\subsection{Algorithm implementation}

In this section, we summarize the algorithm steps and parameters that are required in our joint disparity and illumination variation estimation method.

\section{Inputs:}

- Occluded areas $\mathcal{O}$ estimated from the initial disparity map based on the ordering [41] and border constraints.

- $\left(T_{1}^{(k)}, T_{2}^{(k)}, r^{(k)}\right)$ for $k \in\{1,2,3\}$.

- Minimum and maximum estimates of the fields to be estimated.

- Upper bounds for the constraints. 
Parameter choice:

- For $S_{1}, \omega_{1}=100, \omega_{2}=100$.

- For $S_{2}, S_{3}$ or $S_{4}: \omega_{3}=200, \omega_{4}=200$.

- For $J^{(1)}, J^{(2)}$ and $J^{(3)}: \omega_{5}=\omega_{6}=\omega_{7}=10$.

- $\lambda_{n} \equiv 1.5$.

The weights were chosen so that all the terms in the averaging step have a contribution of the same order.

\section{Stopping criterion:}

- The algorithm is stopped when the following condition is satisfied for 10 successive iterations: $\left\|\mathbf{u}_{n+1}-\mathbf{u}_{n}\right\|<\epsilon\left\|\mathbf{u}_{n}\right\|$ where $\epsilon=10^{-5}$.

Outputs:

- The final values of the disparity vector $\mathbf{u}_{n}$ and the illumination variation vector $\mathbf{v}_{n}$.

\subsection{Gray level images without variation illumination}

Although the purpose of this work is to focus on situations where illumination variation arises, in this first experiment we aim at illustrating the performance of the proposed approach on grayscale images $(K=1)$ without any variation of the illumination field (for every $\mathbf{s} \in \mathcal{A}, v(\mathbf{s})=1)$. This allows us to compare our method with some recent state-of-the-art methods referenced in the Middlebury website ${ }^{2}$. In Table 2, we display the results obtained on 3 different stereo pairs (Teddy, Venus and Cones) for which we compare the results obtained by:

- the correlation-based approach used to initialize our approach (NCC),

- a graph-cut based approach (Gc+Occ) [1],

- a total variation based algorithm (ConvexTV) [42],

- a method based on the knowledge of Ground Control Points (GlobalGCP) [43],

- a histogram-based method (HistoAggr) [44],

- a curvelet-based approach (CurveletSupWgt) [45],

- the approach based on set theoretic estimation (subg. proj.) in [7],

- our proposed method based on PPXA + minimizing an $\ell_{1}$ cost subject to constraints $S_{1,1}, S_{1,2}$ and $S_{1,2}^{\prime}$.

Note that the frame employed to define constraint $S_{1,2}^{\prime}$ simply consists of an overcomplete Haar wavelet representation carried out over 1 resolution level. Other wavelet families have been tested without observing significant improvements.

The proposed approach appears to be competitive with respect to the other ones, although most of them

\footnotetext{
2 http://vision.middlebury.edu/stereo/
}

\begin{tabular}{|l|l|l|l|}
\hline Method & Teddy & Venus & Cones \\
\hline \hline NCC & $1.169^{8}$ & $0.411^{8}$ & $1.134^{8}$ \\
\hline Gc+Occ [1] & $1.166^{7}$ & $0.303^{7}$ & $0.585^{6}$ \\
\hline ConvexTV [42] & $0.366^{1}$ & $0.178^{1}$ & $0.374^{1}$ \\
\hline GlobalGCP [43] & $0.489^{2}$ & $0.272^{4}$ & $0.443^{3}$ \\
\hline HistoAggr [44] & $0.788^{4}$ & $0.278^{5}$ & $0.434^{2}$ \\
\hline CurveletSupWgt [45] & $0.831^{5}$ & $0.299^{6}$ & $0.831^{7}$ \\
\hline Subg. proj. [7] & $0.890^{6}$ & $0.207^{2}$ & $0.551^{5}$ \\
\hline PPXA+ & $0.666^{3}$ & $0.211^{3}$ & $0.487^{4}$ \\
\hline
\end{tabular}

Table 2 Comparative results based on MAE for Teddy, Venus and Cones stereo pairs. The red superscript numbers represent method ranking.

are not able to deal with illumination variation. Moreover, as already mentioned, the proposed method can be implemented on a GPU architecture, thus reducing drastically the computation time. Indeed, the proximity operators and the projections onto the different convex sets can be computed in parallel. For example, with a GPU implementation, when considering the range values $S_{1,1}$ and the total variation constraints $S_{1,2}$, the following executing times per iteration were measured: for Teddy: $21.7 \mathrm{~ms}$, for Venus: $5.72 \mathrm{~ms}$, and for Cones: $21.98 \mathrm{~ms}$.

Let us now turn our attention to more challenging scenarii including illumination variation both for grayscale and color images.

\subsection{Gray level images with illumination variation}

In this section, we evaluate our method on grayscale images $(K=1)$ in the presence of real and simulated illumination changes. The results are obtained on three stereo pairs depicted in Fig. 1, named "Books", "Dolls" and "Parking meter".
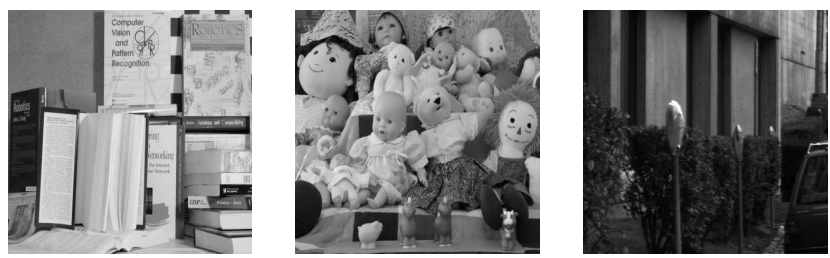

Fig. 1 Stereo images: "Books" (left), "Dolls" (center) and "Parking meter" (right).

In a first experiment, we estimate the disparity map and the illumination variation (real case) on the Books pair downloaded from MiddleBury website. We take here $\phi^{(1)}=|\cdot|$ for the cost function and consider the two convex constraints $S_{1}$ and $S_{2}$ for which the upper bounds were evaluated on the ground truth maps. In 
Fig. 2, we compare our results with the subgradient projection approach proposed in [7], where a strictly convex quadratic function is used. We also compare our approach with two naive procedures for the disparity field estimation: the correlation based approach ignoring the illumination variation and a histogram equalization technique followed by a variational approach for disparity estimation, in the spirit of the approach we developed.

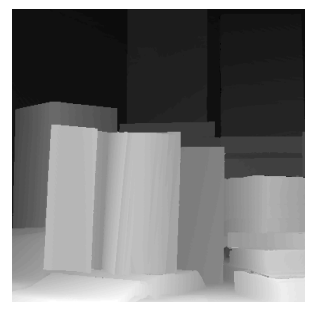

a) True disparity

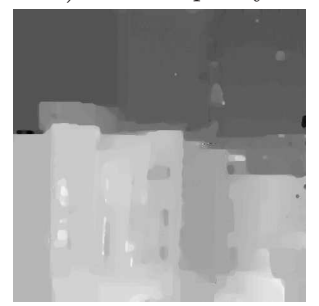

c) $\mathrm{MAE}=1.8, \operatorname{Err}=16$

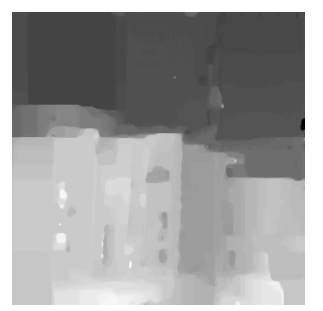

e) $\mathrm{MAE}=1.46, \mathrm{Err}=13$

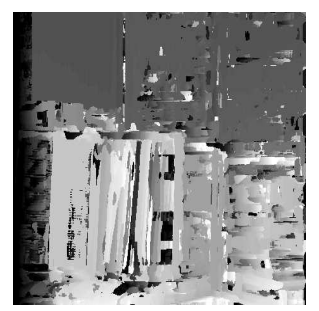

g) $\mathrm{MAE}=4.69, \mathrm{Err}=26$

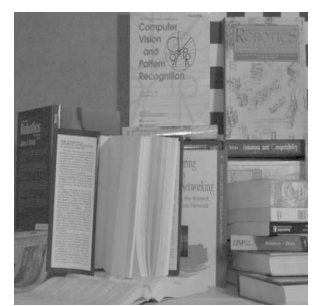

b) True illumination

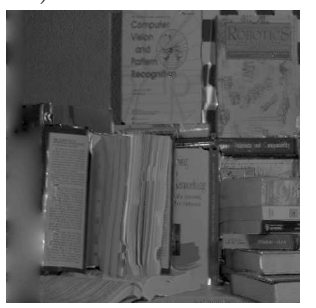

d) $\mathrm{MAE}=0.212$

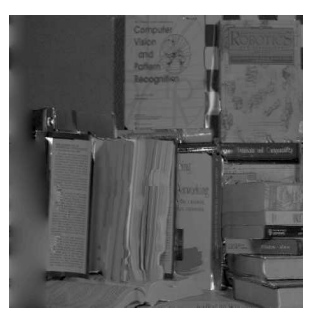

f) $\mathrm{MAE}=0.211$

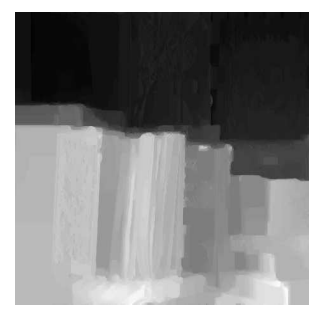

h) $\mathrm{MAE}=1.55, \mathrm{Err}=18$
Fig. 2 Results for "Books" stereo pair: a)-b) ground truths, c)d) subgradient projection method (c) disparity and d) illumination fields) and e)-f) proposed approach with $\left(u_{\min }, u_{\max }\right)=$ $(20,75),\left(v_{\min }, v_{\max }\right)=(0.1,1.1),\left(\tau_{2}, \kappa_{2}\right)=(74000,400)(\mathrm{e})$ disparity and f) illumination fields), g) estimation of the disparity by $\mathrm{NCC}, \mathrm{h}$ ) estimation of the disparity after histogram equalization.

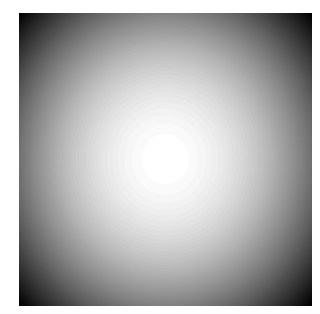

Fig. 3 Synthetic Gaussian profile.

Concerning the choice of the criterion and of the constraint sets, we present in Fig. 4 results obtained by using the following settings:

- for a)-b): $S_{1}, S_{2}^{\prime}$, and $\phi^{(1)}=|\cdot|$;

- for c)-d) same constraints as a)-b) and $\phi^{(1)}=(\cdot)^{4}$;

- for e)-f): $S_{1}, S_{2}, S_{3}$, and $\phi^{(1)}=|\cdot|$;

- for $\mathrm{g}$ )-h) same constraints as e)-f) and $\phi^{(1)}=|\cdot|^{\frac{3}{2}}$.

It turns out that the $\ell_{1}$ cost is a good choice, and that the use of second-order constraints leads to a marginal improvement.

In a third experiment (see Fig. 5), we show the results provided by the proposed method $\left(\phi^{(1)}=|\cdot|\right)$ for a real image stereo pair from JISCT database ${ }^{3}$, taken under simulated illumination variation. To introduce a significant illumination variation, the left image is kept untouched and we modified the right image by multiplying it with the Gaussian profile $g$ depicted in Fig. 3 and defined as:

$$
\begin{aligned}
& (\forall(x, y) \in \mathcal{A}) \\
& g(x, y)=\alpha_{1} \exp \left(-\frac{\left(x-x_{0}\right)^{2}+\left(y-y_{0}\right)^{2}}{2 \xi^{2}}\right)+\alpha_{2}
\end{aligned}
$$

where $\left(x_{0}, y_{0}\right)$ is the image center, $\xi=512$ is the illumination deviation, $\alpha_{1}$ and $\alpha_{2}$ are given constants equal to 1.8 and -0.6 respectively.

In this case, the constraint bounds in $S_{1}$ and $S_{2}$ are not known. Hence, they were firstly computed on the initial disparity and then divided by a factor 2 , thus assuming that the expected result should be smoother than the initial estimate.

\subsection{Color images with illumination variation}

For color images, we present tests performed on Dolls pair also downloaded from Middlebury website. Constraint sets $S_{1}$ and $S_{2}$ were used, the associated bounds being computed on the ground truth fields. The results are provided in Fig. 6 for YUV color images and the grayscale level images ( $Y$ component only) by using, for

\footnotetext{
3 http://vasc.ri.cmu.edu/idb/html/jisct/
} 


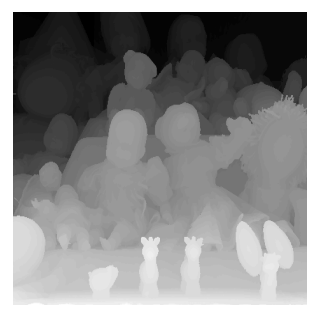

a) True disparity

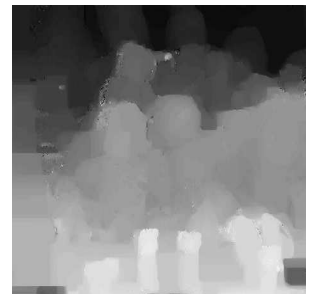

c) $\mathrm{MAE}=\overline{1.26}, \mathrm{Err}=13$

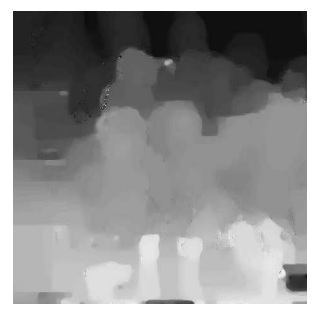

e) $\mathrm{MAE}=1.38, \operatorname{Err}=14$

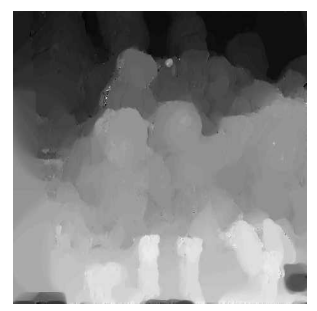

g) $\mathrm{MAE}=1.23, \mathrm{Err}=14$

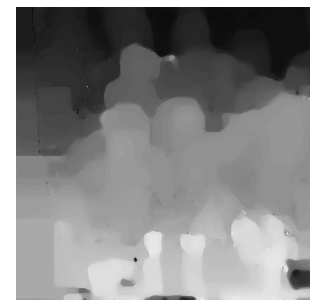

i) $\mathrm{MAE}=1.23, \mathrm{Err}=14$

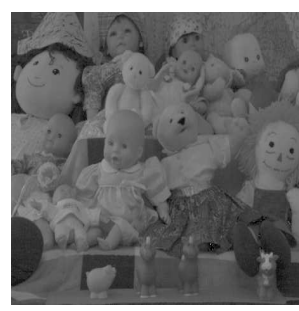

b) True illumination

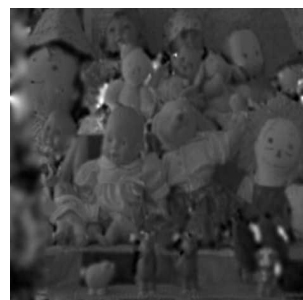

d) $\mathrm{MAE}=0.09$

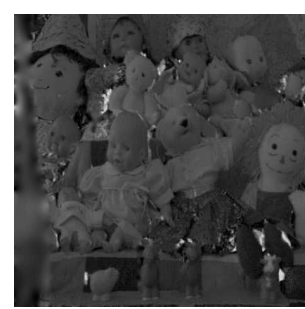

f) $\mathrm{MAE}=0.102$

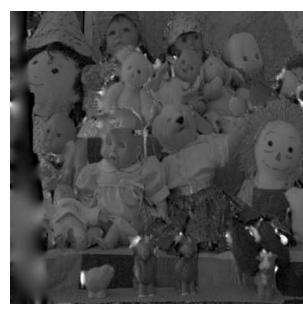

h) $\mathrm{MAE}=0.103$

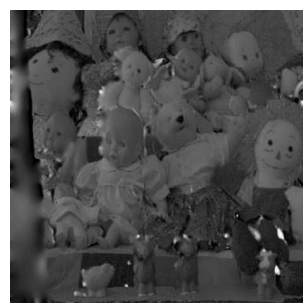

j) $\mathrm{MAE}=0.103$

Fig. 4 Results for Dolls stereo pair: proposed approach considering for c)-d)- $\ell_{1}$-cost and $S_{1} \cap S_{2}^{\prime}$ with $\left(u_{\min }, u_{\max }\right)=$ $(20,75),\left(v_{\min }, v_{\max }\right)=(0.1,1.1),\left(\tau_{2}^{\prime}, \kappa_{2}\right)=(92000,230)$, e)f) $-\ell_{4}$ cost and $S_{1} \cap S_{2}^{\prime}$, g)-h) $\ell_{1}$ cost and $S_{1} \cap S_{2} \cap S_{3}$ with $\left.\left.\left(\tau_{2}, \kappa_{2}\right)=(34000,230),\left(\tau_{3}, \kappa_{3}\right)=(180000,570), \mathrm{i}\right)-\mathrm{j}\right)-\ell_{3} / 2$ cost and $S_{1} \cap S_{2} \cap S_{3}$

every $k \in\{1, \ldots, K\}, \phi^{(k)}=|\cdot|$. As expected, matching errors are reduced by using the proposed illumination variation model for color images.

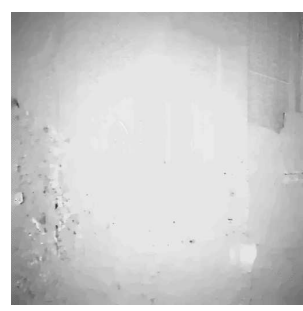

a) $\mathrm{MAE}=0.033$

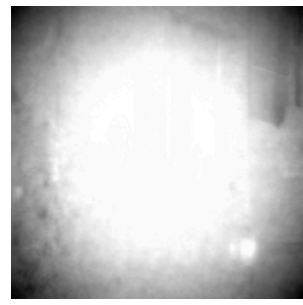

c) $\mathrm{MAE}=0.031$

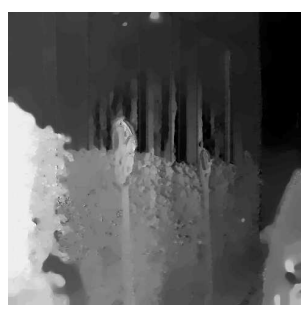

b)

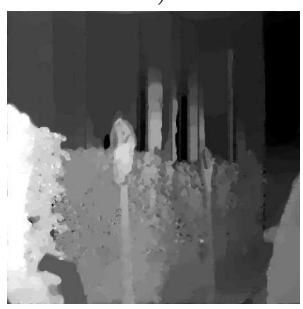

d)
Fig. 5 Results for "Parking meter" stereo pair. a)-b) Subgradient projection method and c)-d) our approach with $\left(u_{\min }, u_{\max }\right)=$ $(1,8),\left(v_{\min }, v_{\max }\right)=(0.1,1.1),\left(\tau_{2}, \kappa_{2}\right)=(20000,0.4)$.

\section{Conclusion}

In this paper, we have investigated the application of a parallel proximal algorithm to the dense disparity estimation problem for multicomponent (e.g. color) stereo matching under illumination variation conditions. The proposed approach is flexible as it allows us to consider various convex objective functions and constraints. It is also able to exploit the potentials offered by multicore/GPU parallel architectures. However, one of the current limitations of the proposed method is that it is limited to convex cost functions, thus requiring a linearization of the original disparity model and making the estimation potentially sensitive to high values of matching errors. In our future work, we therefore plan to consider extensions of this approach to nonconvex cost functions.

Acknowledgements We would like to thank Prof. Wided Miled for providing us with her codes. We would also like to thank Dr. Raffaele Gaetano and Giovanni Chierchia for their implementation of our approach on GPU architectures.

\section{References}

1. V. Kolmogorov and R. Zabih. Computing visual correspondence with occlusions using graph cuts. In Proc. IEEE Int. Conf. Comput. Vis., volume 2, pages 508-515, Vancouver, BC, Canada, Jul. 9-12, 2001.

2. Y. Boykov, O. Veksler, and R. Zabih. Fast approximate energy minimization via graph cuts. IEEE Trans. Pattern Anal. Mach. Intell., 23(11):1222-1239, 2011.

3. A. Klaus, M. Sormann, and K. Karner. Segment-based stereo matching using belief propagation and a self-adapting dis- 


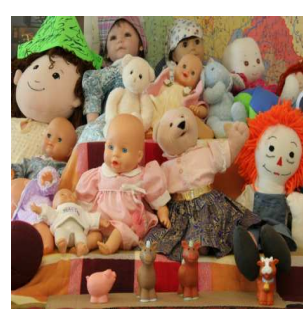

a) Left image

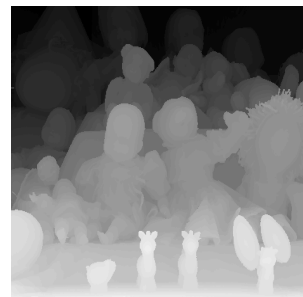

c) True disparity

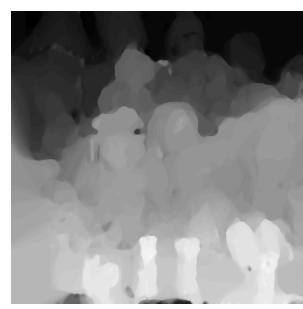

e) $\mathrm{MAE}=1.26, \mathrm{Err}=13$

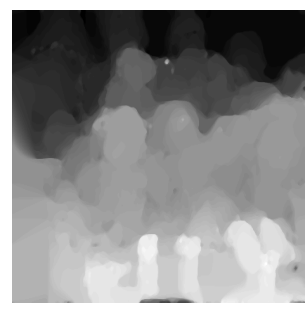

g) $\mathrm{MAE}=1.10, \operatorname{Err}=11$

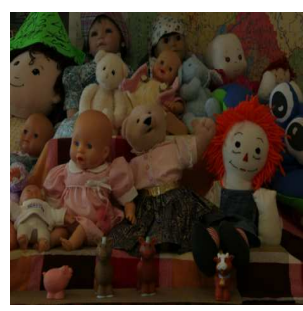

b) Right image

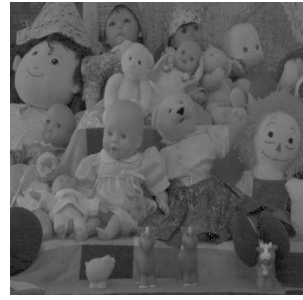

d) True illumination

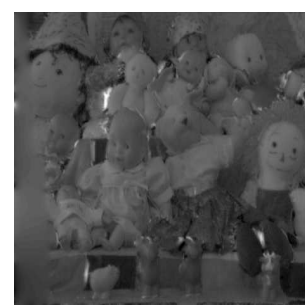

f) $\mathrm{MAE}=0.09$

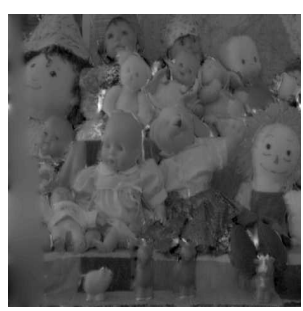

h) $\mathrm{MAE}=0.09$
Fig. 6 Results for Dolls stereo pair: e)-f) results from gray scale images and $\mathrm{g})-\mathrm{h})$ from color (YUV) images. $\left(\left(u_{\min }, u_{\max }\right)=\right.$ $\left.(20,75),\left(v_{\min }, v_{\max }\right)=(0.1,1.1),\left(\tau_{2}, \kappa_{2}\right)=(92000,240)\right)$.

similarity measure. In Proc. Int. Conf. Pattern Recogn., volume 3, pages 15-18, Hong Kong, Aug., 20-24 2006.

4. Q. Yang, L. Wang, R. Yang, S. Wang, M. Liao, and D. Nistér. Realtime global stereo matching using hierarchical belief propagation. In Proc. British Machine Vision Conference, pages 989-998, Edinburgh, U.K, Sep. 4-7 2006.

5. R. Deriche, P. Kornprobst, and G. Aubert. Optical-flow estimation while preserving its discontinuities: A variational approach. In S. Li, D. Mital, E. Teoh, and H. Wang, editors, Recent Developments in Computer Vision, volume 1035 of Lecture Notes in Computer Science, pages 69-80. Springer Berlin / Heidelberg, 1996.

6. W. Miled, J.-C. Pesquet, and M. Parent. Disparity map estimation using a total variation bound. In The 3rd Canadian Conf. on Computer and Robot Vision, pages 48-55, Quebec, Canada, Jun. 2006.

7. W. Miled, J.-C. Pesquet, and M. Parent. A convex optimisation approach for depth estimation under illumination variation. IEEE Trans. Image Process., 18(4):813-830, Apr. 2009.

8. I. J. Cox, S. Roy, and S. L. Hingorani. Dynamic histogram warping of image pairs for constant image brightness. In Proc. Int. Conf. Image Process., volume 2, pages 366-369, Washington, DC, Oct. 23-26 1995.

9. R. Zabih and J. Woodfill. Non-parametric local transforms for computing visual correspondence. Proc. Eur. Conf. Comput. Vis., pages 15-158, Stockholm, Sweden, May 2-6 1994.

10. J. E. Davis, R. Yang, and L. Wang. BRDF invariant stereo using light transport constancy. In Proc. IEEE Int. Conf. Comput. Vis., volume 1, pages 436-443, Beijing, China, Oct. 15-21 2005.

11. M.A. Gennert. Brightness-based stereo matching. In Proc. IEEE Int. Conf. Comput. Vis., pages 139-143, Tampa, FL, Dec. 5-8 1988.

12. J.-C. Pesquet and N. Pustelnik. A parallel inertial proximal optimization method. Pac. J. Optim., 2012. accepted, http://www.optimizationonline.org/DB FILE/2010/11/2825.pdf.

13. A. Fusiello, E. Trucco, and A. Verri. A compact algorithm for rectification of stereo pairs. Mach. Vis. Appl., 12(1):16-22, 2000.

14. D. Tsai, C. Lin, and J. Chen. The evaluation of normalized cross correlations for defect detection. Pattern Recogn. Lett., 24(15):2525-2535, 2003.

15. L. I. Rudin, S. Osher, and E. Fatemi. Nonlinear total variation based noise removal algorithms. Phys. D, 60:259-268, 1992.

16. P. L. Combettes and J.-C. Pesquet. Image restoration subject to a total variation constraint. IEEE Trans. Image Process., 13(9):1213-1222, Sep. 2004.

17. P. L. Combettes and J.-C. Pesquet. A proximal decomposition method for solving convex variational inverse problems. Inverse Problems, 24(6): $\mathrm{x}+27$, Dec. 2008.

18. N. Pustelnik, C. Chaux, and J.-C. Pesquet. Parallel ProXimal algorithm for image restoration using hybrid regularization. IEEE Trans. Image Process., 20(9):2450 -2462, Nov. 2011.

19. J. F. Aujol, G. Aubert, L. Blanc-Féraud, and A. Chambolle. Image decomposition into a bounded variation component and an oscillating component. J. Math. Imaging Vision, 22(1):71-88, 2005.

20. A. Chambolle, R. A. DeVore, N.-Y. Lee, and B. J. Lucier. Nonlinear wavelet image processing: variational problems, compression and noise removal through wavelet shrinkage. IEEE Trans. Image Process., 7:319-335, Mar. 1998.

21. D. Han and D. R. Larson. Frames, bases, and group representations. In Mem. Amer. Math. Soc., volume 147, pages $\mathrm{x}+94$. AMS, 2000.

22. S. Mallat. A Wavelet Tour of Signal Processing. Academic Press, San Diego, USA, 1997.

23. S. Lefkimmiatis, A. Bourquard, and M. Unser. Hessian-based norm regularization for image restoration with biomedical applications. IEEE Trans. Image Process., 21(3):983-995, Mar. 2012.

24. J. J. Moreau. Fonctions convexes duales et points proximaux dans un espace hilbertien. C. R. Acad. Sci., 255:2897-2899, 1962.

25. P. L. Combettes. The foundations of set theoretic estimation. Proc. IEEE, 81(2):182-208, Feb. 1993.

26. P. L. Combettes and J.-C. Pesquet. Proximal splitting methods in signal processing. In H. H. Bauschke, R. Burachik, P. L. Combettes, V. Elser, D. R. Luke, and H. Wolkowicz, editors, Fixed-Point Algorithms for Inverse Problems in Science and Engineering, pages 185-212. Springer-Verlag, New York, 2011. 
27. D. C. Youla and H. Webb. Image restoration by the method of convex projections: Part I - theory. IEEE Trans. Med. Imag., 1(2):81-94, Oct. 1982.

28. E. van den Berg and M. P. Friedlander. Probing the Pareto frontier for basis pursuit solutions. SIAM J. Sci. Comput., 31(2):890-912, Nov. 2008.

29. Y. C. Eldar and M. Mishali. Robust recovery of signals from a structured union of subspaces. IEEE Trans. Inf. Theory, 55(11):5302-5316, Nov. 2009.

30. M. Afonso, J. Bioucas-Dias, and M. A. T. Figueiredo. An augmented Lagrangian approach to the constrained optimization formulation of imaging inverse problems. IEEE Trans. Image Process., 20(3):681-695, Mar. 2011.

31. S. Setzer, G. Steidl, and T. Teuber. Deblurring Poissonian images by split Bregman techniques. J. Vis. Comm. Image Repr., 21:193-199, 2010.

32. H. Attouch and M. Soueycatt. Augmented Lagrangian and proximal alternating direction methods of multipliers in Hilbert spaces - applications to games, PDE's and control. Pac. J. Optim., 5(1):17-37, Jan. 2009.

33. G. Chen and M. Teboulle. A proximal-based decomposition method for convex minimization problems. Math. Program., 64(1):81-101, 1994. Ser. A.

34. A. Chambolle and T. Pock. A first-order primal-dual algorithm for convex problems with applications to imaging. $J$. Math. Imaging Vision, 40(1):120-145, 2011.

35. E. Esser, X. Zhang, and T. Chan. A general framework for a class of first order primal-dual algorithms for convex optimization in imaging science. SIAM J. Imaging Sci., 3(4):1015-1046, 2010.

36. P. L. Combettes, Đinh Dũng, and B. C. Vũ. Proximity for sums of composite functions. J. Math. Anal. Appl., 320(2):680-688, Aug. 2011.

37. L. M. Briceño-Arias and P. L. Combettes. A monotone + skew splitting model for composite monotone inclusions in duality. SIAM J. Optim., 21(4):1230-1250, Oct. 2011.

38. P. L. Combettes and J.-C. Pesquet. Primal-dual splitting algorithm for solving inclusions with mixtures of composite, lipschitzian, and parallel-sum type monotone operators. SetValued Var. Anal., 2012. 22 pp.

39. N. Pustelnik, J.-C. Pesquet, and C. Chaux. Relaxing tight frame condition in parallel proximal methods for signal restoration. IEEE Trans. Signal Process., 60(2):968-973, Feb. 2012.

40. K.-J. Yoon and I.-S. Kweon. Adaptive support-weight approach for correspondence search. IEEE Trans. Pattern Anal. Mach. Int., 28(4):650-656, Apr. 2006.

41. A. L. Yuille and T. Poggio. A generalized ordering constraint for stereo correspondence. Technical Report AIM-r77, 1984.

42. T. Pock, T. Schoenemann, G. Graber, H. Bischof, and D. Cremers. A convex formulation of continuous multilabel problems. In Proc. Eur. Conf. Comput. Vis., pages 792-805, Marseille, France, Oct. 2008.

43. L. Wang and R. Yang. Global stereo matching leveraged by sparse ground control points. In Proc. IEEE Conf. Comput. Vis. and Patt. Rec., pages 3033-3040, Colorado Springs, USA, Jun. 20-25 2011.

44. D. Min, J. Lu, and M. Do. A revisit to cost aggregation in stereo matching: how far can we reduce its computational redundancy? In Proc. IEEE Int. Conf. Comput. Vis., pages 1567-1574, Barcelona, Spain, Nov. 2011.

45. D. Mukherjee, G. Wang, and J. Wu. Stereo matching algorithm based on curvelet decomposition and modified support weights. In Proc. Int. Conf. Acoust., Speech Signal Process., pages 758-761, Dallas, Texas, USA, Mar. 14 - 192010. 